\title{
Tracking Technical Refinement in Elite Performers: The Good, the Better, and the Ugly
}

\author{
Howie J. Carson \\ University of Central Lancashire \\ Dave Collins \\ University of Central Lancashire
}

\begin{abstract}
This study extends coaching research examining the practical implementation of technical refinement in elite-level golfers. In doing so, we provide an initial check of precepts pertaining to the Five-A Model and, examine the dynamics between coaching, psychomotor, biomechanical, and psychological inputs to the process. Three case studies of golfers attempting refinements to their already well-established techniques are reported. Kinematic data were supplemented with intraindividual movement variability and self-perceptions of mental effort as measures of tracking behavior and motor control. Results showed different levels of success in refining technique and subsequent ability to return to executing under largely subconscious control. In one case, the technique was refined as intended but without consistent reduction of conscious attention, in another, both were successfully apparent, whereas in the third case neither was achieved. Implications of these studies are discussed with reference to the process' interdisciplinary nature and importance of the initial and final stages.
\end{abstract}

Keywords: case studies; focus of attention; golf coaching practice; movement variability; technical change; the Five-A Model.

Contrary to finite stage models proposed by skill acquisition theory (Bernstein, 1967; Fitts \& Posner, 1967; Gentile, 1972), the reality of applied motor control for elite performers (i.e., those at the skill stabilization, autonomous, or fixation/ diversification stage) and their coaches, shows that skill development does not terminate once a final stage has been achieved (Carson, Collins, \& MacNamara, 2013; Schack \& Bar-Eli, 2007; Toner \& Moran, 2015). Rather, for these perform$\mathrm{ers}^{1}$, the nature and level of challenges faced are distinct from those of an initial

The authors are with the Institute of Coaching and Performance, University of Central Lancashire, Preston, UK. Address author correspondence to Howie Carson at HCarson1@uclan.ac.uk. 
stage learner. One such challenge relates to the implementation and optimization of skill refinement, whereby a new, modified version of a previously acquired and well-established technique must remain permanent and consistent when performed (cf. MacPherson, Collins, \& Obhi, 2009; Wood \& Wilson, 2011); in short, execution must be pressure resistant (Carson \& Collins, 2011). Recognizing these unique and important requirements serves to highlight a significant gap within the literature, thus establishing and delineating between two separate processes, skill acquisition and refinement. Consequently, there is a need for inquiry to understand what makes these processes distinct and, how optimal solutions may be delivered by coaches and applied sport psychology/science support practitioners.

Reflecting this gap, effective systems for skill refinement also appear to be insufficiently considered within applied practice. Therefore, at present, such a task comes with a certain degree of risk (see also our General Discussion section), both in terms of skill and career outcome (cf. golfers Ian Baker-Finch, Craig Perks, and David Duval). For example, Carson et al. (2013) reported frequent cases from European Tour golfers and coaches of almost continuous refinement to prevent or remedy regression toward an undesirable previous technique; therefore highlighting a common problem of nonpermanency at this high level. Overall, this study discovered the inconsistent use of processes employed at both inter- and intraindividual levels. In addition, when reporting individual cases of what were considered to be successful technical refinement (as understood by self-prescribed criteria), participants often reported subsequent failures when attempting to execute their new technique in competitive situations, demonstrating a lack of proactive pressure resistant practices. What is clear from these exemplar cases are problems relating to practitioners not knowing that effective systems for skill refinement are needed, how to do it, criteria for knowing when to stop refining/when refinement is complete, and how to promote pressure resistance before competitive reintroduction. As such, there is a clear and current need within golf (as an exemplar for other sports, especially closed and self-paced skills) to explore the development and testing of systematic models to facilitate permanent and pressure resistant skill refinement.

Addressing this gap from a theoretical perspective, Carson and Collins (2011) proposed the systematic Five-A Model based on the existent literature. Central to its suggestions is the combined use of motor control, sport psychology, and coaching principles, presenting an interdisciplinary five-stage guide for applied practice. To summarize these stages, skill refinement must be preceded by a detailed process of "Analysis" in which both performer and coach are actively involved, followed by necessary stages of "Awareness" whereby the skill is deautomated, "Adjustment" then "(Re)Automation" of the skill. Finally, a series of proactive steps must be taken to increase resistance against the negative effects of competitive pressure within the "Assurance" stage (for a detailed account of this model and its informing literature, see Carson \& Collins, 2011, 2014).

From an empirical perspective, sport psychology and motor control research has provided useful insights to support the suggestions of Carson and Collins (2011), offering potential tracking measures throughout the Five-A Model. For instance, experimental studies have shown intraindividual movement variability and performers' perceptions of mental effort (conscious processing) as related (Carson, 
Collins, \& Richards, 2014a; MacPherson, Collins, \& Morriss, 2008). Specifically, a narrow internal part-skill focus of attention (i.e., Awareness stage) resulted in lower intertrial variability for that component, while the variability of other unrelated technical components increased slightly. Paradoxically, when performers reduced their focus toward that movement component and adopted a more holistic focus of attention (i.e., (Re)Automation stage), variability levels were shown to be more consistent across the different components of technique. Therefore, movement variability combined with perceptual data of mental effort could potentially provide valid measures to reflect performers' level of automated control (Carson \& Collins, 2016) throughout the Five-A Model (see Carson, Collins, et al., 2014a, Figure 1 for a representation of this process in its entirety). Moreover, being able to assess a performer's level of mental effort through accurate means other than self-report, offers a beneficial "tool" for coaches, since an optimal outcome would seek to avoid excessive attention toward the technique (cf. MacPherson et al., 2008).

Crucially, however, these control measures must also be supported by objective indicators of behavior, for example, using kinematic analyses to track the refinement, if we are to have certainty in their veracity (Carson \& Collins, 2014). Advances in motion capture modeling, using six degrees-of-freedom and a segment co-ordinate system, have been shown to reveal most accurately the exact movements of interest (Brown, Selbie, \& Wallace, 2013), therefore affording a comprehensive understanding of the technique. Such an addition is also essential to offering a multilevel approach (cf. Newell, Liu, \& Mayer-Kress, 2001) to understanding the process of technical refinement in applied coaching environments.

Therefore, reflecting these theoretical, empirical, and applied considerations, this study aimed to provide an initial check of precepts pertaining to the Five-A Model and examine the dynamic interaction between coaching, psychomotor, biomechanical, and psychological inputs to the skill refinement process. To most effectively highlight these relationships, we present three contrasting case studies of elite-level golfers undertaking technical refinements. Notably, each provide different outcomes: "good" (somewhat successful), "better" (very successful) and "ugly" (unsuccessful). Accordingly, we now offer a general methodological overview, followed by separate reporting of the individual case studies.

\section{General Methodology}

\section{Participants}

Three right-handed male English golfers took part in this study. Reflecting their elite skill status, John (pseudonym), was a 42 year old PGA Professional with a previous handicap of +3 and 32 years of playing experience, Chris (pseudonym), was a 27 year old professional golfer on the PGA Europro Tour with a previous handicap of +1 and 20 years of playing experience, and Peter (pseudonym), was a 25 year old amateur golfer with a handicap of 0 and 18 years of playing experience. Ethical approval was granted from the university's ethics committee and informed consent was obtained from all participants before data collection. 


\section{Data Collection}

Data were collected on the outdoor practice facility of each participant's golf club, either in front of a practice net or on the driving range (depending on the practice being performed) and between one-three times per week. A data collection session required 10 shots with participants' own 7-iron. A 7-iron was selected because each refinement related to the full swing; this club was considered by all as frequently used and would, therefore, most likely be well-established and contain the technical error.

Three-dimensional kinematic data were collected using a mobile inertial sensor motion capture suit (MVN Biomech Suit, Xsens Technologies B.V., The Netherlands), as described by Carson, Collins, and Richards (2014b). Following each 10 trials, participants described the direction and intensity of their attentional focus; which required a self-report protocol as described by Carson, Collins, et al. (2014a) using the Rating Scale for Mental Effort (RSME; Zijlstra, 1993). As an informal aspect of the refinement process, however, and a standard element of professional practice and development (cf. A. Martindale \& Collins, 2007), reflective field notes were kept by the corresponding author pertaining to behavioral observations and self-reports by the participants, when considered as potentially beneficial to interpreting data collected.

\section{Data Processing and Analysis}

Raw data from the MVN Studio Software (Xsens Technologies B.V., The Netherlands) were exported into c3d file format and analyzed using six degrees-of-freedom modeling with Visual3D v4.89.0 software (C-Motion Inc., USA). Swing events were identified to enable consistent extraction of kinematic and variability (standard deviation) data pertaining to participants' intended technical refinement (target variable) and unrelated (nontarget variable) kinematics. Whereas target variables could be identified from self-reports and an evident reduction in variability (in most cases) following initial sessions, the nontarget variable could only be determined retrospectively after several more sessions; since there was little way of knowing exactly what variable would be unrelated at the level of central nervous system organization. Kinematic and variability data were exported to Microsoft Excel 2010 and plotted graphically with trend lines added using the software's linear function.

Due to the individual nature of these data, statistical treatment was applied on a case-by-case basis to supplement visual inspection of the graphs. The splitmiddle technique (White, 1974) was implemented across stages of interest to assess changes in level (mean value) and trend line slope, as described by Marlow, Bull, Heath, and Shambrook (1998). In this analysis, increases are indicated by a " $X$ " sign and decreases by a " $\div$ " sign. Statistical differences between these data were assessed using Binomial tests, with the level of significance set at 0.05 . Using the calculations reported by Callow, Hardy, and Hall (2001), these tested the null hypothesis that there was no difference between stages by analyzing the number of data points above (or below) an extended trend line between stages. Significant differences would therefore suggest that the training had caused a difference in the variable being analyzed. Such analyses are common when conducting single 
case study research (e.g., Mesagno \& Mullane-Grant, 2010; Shambrook \& Bull, 1996) and can enhance objectivity when compared with visual inspection alone.

\section{Case Study 1: John "the Good"}

\section{Background of Individual and Technique}

Following the season's (April-October) end, John decided to undergo a selfdiagnosed refinement which was contemplated some months earlier. During consultation, John explained how he had already started to work on the refinement, which required repositioning the left elbow in a downward direction during the backswing, as opposed to pointing away from his body and toward the golf ball (as he described). John's rationale was to steepen his golf club swing plane and, in turn, reduce his ball flight curvature. As such, in a manner not untypical of some player-coach relationships, prechange data were unable to be collected. This case study did not, therefore, represent a complete account of the Five-A Model. The intervention's main focus was to refine John's technique using a process of initially high and then, through gradual reduction, lower levels of mental effort directed toward the target variable, informed by the tracking measures described earlier.

\section{Procedures Used in Case Study 1}

Since John had not considered the type of practice that would encourage increased conscious control (cf. Bjork \& Bjork, 2011), practice began on the driving range; however, after approximately two weeks John reported that his swing had not changed and wanted to seek advice on how to resolve this. Advice was provided to practice four times per week using short, random, and distributed sessions in front of a net. Executions from in front of a net were intended to lessen the potential for distraction from task-irrelevant outcome stimuli and enhance his self-directed attention toward the target variable. Indeed, this decision was informed by evidence that information is more accurately retrieved from memory when cognition is separated from environmental influence; a strategy referred to as gaze aversion when contextualized within everyday memory tasks (e.g., looking toward the sky or floor to recall a telephone number; Carson, Collins, \& Richards, 2015; Glenberg, Schroeder, \& Robertson, 1998). Reassurance that shot outcome was not important, so long as kinaesthetic control increased over achieving the new position, was an important feature at this stage (cf. Christina \& Corcos, 1988). It was also explained that this more "difficult" practice would help John to realize and later internalize the refinement in a way that would increase transferability across different playing conditions.

As the technique began to change, John was instructed to gradually taper out his practice sessions from the net and begin to practice on the driving range $(3: 1$, $2: 2,1: 3,0: 4)$ while incorporating a holistic rhythm-based cue (e.g., "swish") in the latter training environment. Following initial success at consciously executing the new elbow position, an immediate return to high-level automaticity was prevented to allow the additional technical components to "settle in" with the newly refined 
technique (cf. Carson \& Collins, 2016). Accordingly, random practice (including nine holes occasionally on the golf course) was still advised in a manner that would prevent John from executing under a low level of mental effort. Practice on the golf course would provide greater familiarization of the new routine while using the holistic rhythm-based cue.

\section{Data Analysis of Case Study 1}

The target variable was defined by referencing the left elbow position to the sternum in the anterior-posterior axis, the nontarget variable as the right elbow position to the sternum in the medial-lateral axis. Data $\left(N_{\text {trials }}=400\right)$ were taken at a "midbackswing" event, defined as the frame when the left hand crossed a threshold of $0.0 \mathrm{~m}$ relative to a predetermined position on the spine (VT12L3) in the vertical axis on swing ascent.

\section{Results of Case Study 1}

During the "high mental effort" stage (Figure 1A), mental effort ratings were very high $($ maximum $=147$, level $=127.58)$, before reducing from Day 47 to a rating as low as 20 in the "low mental effort" stage (level = 38.44). Between Days 9-14 mental effort decreased, before John applied greater attention to the target variable; representing the transition from practice on the driving range to in front of the net (slope $=0.042$ ). Despite an expected overall decline in mental effort during the low mental effort stage (slope $=-0.82$ ), John reported small but frequent increases; however, a significant difference was still evident, $p<.001$.

Kinematic data showed the target variable reducing in distance as per John's intended refinement (Figure 1B). On Day 1 the mean distance was $20.15 \mathrm{~cm}$; however, this reduced to $14.94 \mathrm{~cm}$ by Day 75 , with a decrease in level from 17.34-15.13 $\mathrm{cm}$. During the first 12 days and low mental effort stage, the technique remained largely unchanged between sessions; John was more consistent from session-tosession. The change in trend line slope between stages $(-0.07$ to 0.005$)$ supports this observation; however, there was no significant difference, $p=.12$.

Upon visual inspection, the contrasting patterns of variability somewhat support the covariance interaction predicted. For the target variable (Figure 1C), despite a decreasing variability trend (slope $=-0.013)$, the level increased $(0.87-1.04)$ between intervention stages. There was minimal difference in slope $(-0.014)$; however, statistical analysis showed a significant difference, $p<.001$, adding support to a change in tendency for conscious control. By contrast, the nontarget variable produced the reverse effect (Figure 1D), an initial increase then decrease in variability; this time with little change in level (1.37-1.33), but almost equal and opposite slope gradients (0.03 to -0.04). A significant difference between the high and low mental effort stages, $p=.002$, further supports the predicted pattern of change. Notably, variability changed in the expected directions for both target and nontarget variables once John began practicing with reduced outcome feedback (i.e., hitting into the net). Thus the kinematic data offer some interesting clues as to optimum practice when refining technique. 


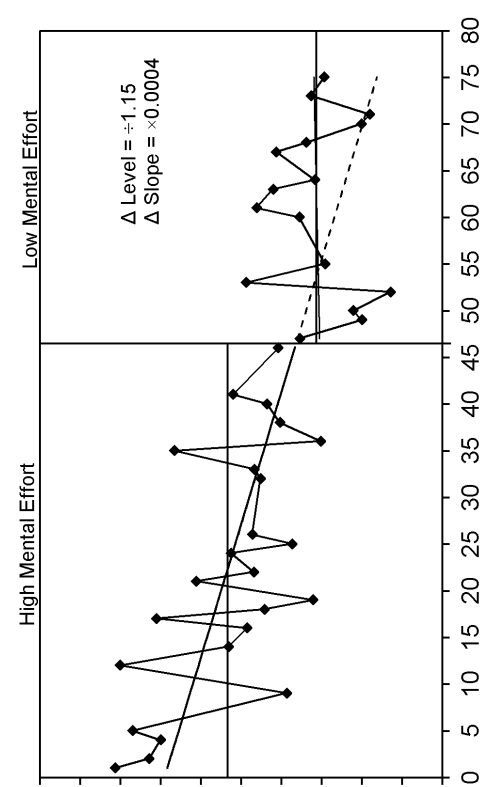

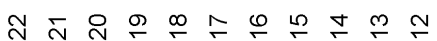

m

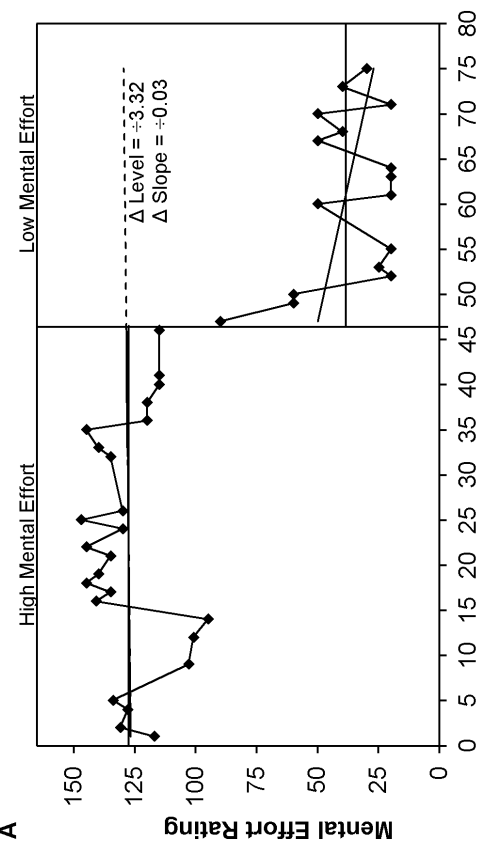

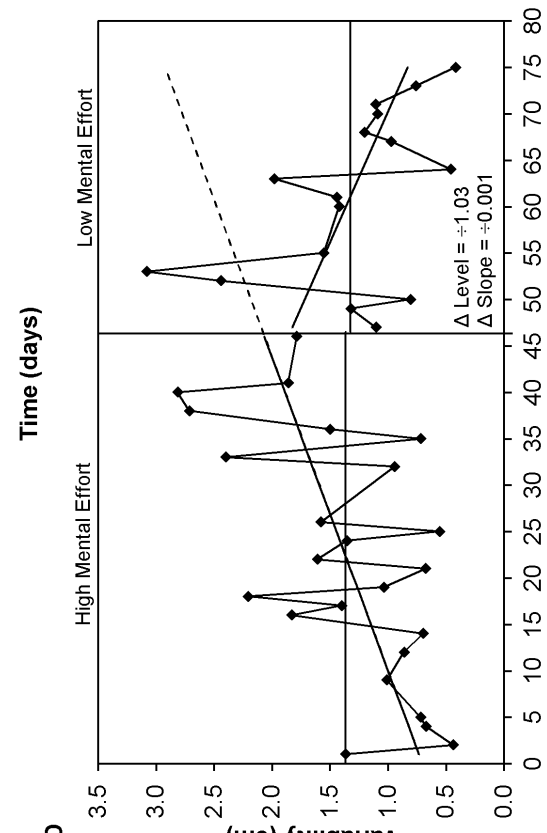

อ

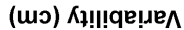

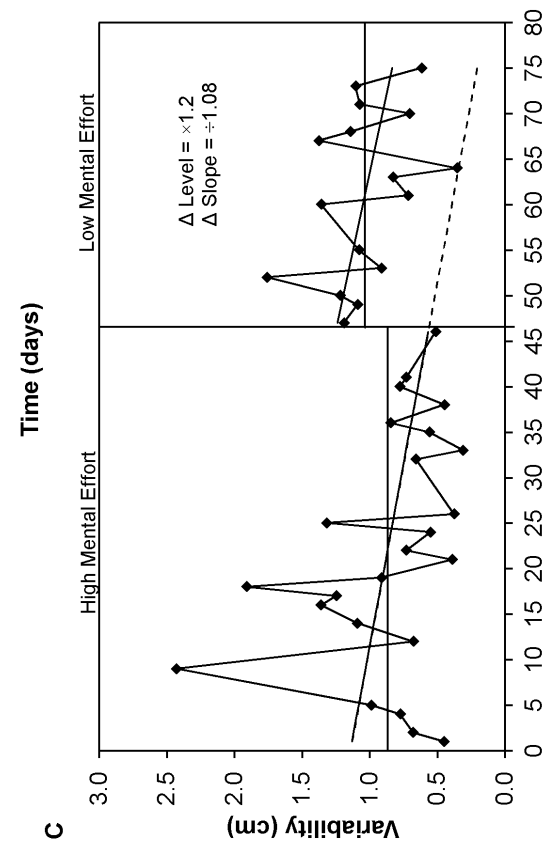

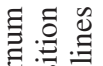

बै

욤

흥 흥

o w :

눙

宅命

를 छ

⿹

응 ज要

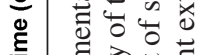

클 잉

0)

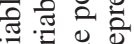

है

च च

$\exists$.

ฮิ)

\pm 0 के

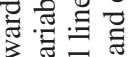

है तี

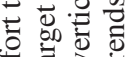

娄 $>$

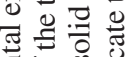

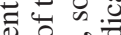

ฮ

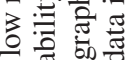

릌킄

穴

की

op $\frac{0}{0} \overline{0}$

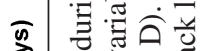

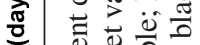

定

of $\bar{E}: \bar{\Xi}$

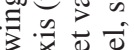

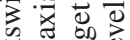

赔

०

.

\&

들

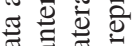

元

跣

$1 . \Xi \frac{\pi}{5}$

๘ $\Xi ี$

는 0

可司

는 0 递 


\section{Brief Discussion of Case Study 1}

Addressing the target variable kinematics, this case study was successful due to achievement of the intended refinement, although no follow up data were collected to assess long-term permanency, nor pressure resistance. Regarding the insignificant Binomial test result, clearly data were influenced by the four initial trials following Day 46 (Figure 1B). Indeed, from Day 53 the majority of data points were above the predicted trend line. As such, we acknowledge the role that statistical analyses serve in supplementing visual inspection; nevertheless, considering the crude method of calculation, common sense or meaningful real-world observations should prevail.

Kinematic variability data provide tentative, yet insightful, preliminary evidence to confirm longitudinal predictions during the refinement process (cf. Carson, Collins, et al., 2014a). A clear feature of these data are the nonlinearity between sessions; although trends are apparent, progress is not smooth. Distinctly, the target variable demonstrated a second reduction on Day 61 indicating that, despite successful change of kinematics, John's control over the target variable was more conscious than preferred at times during this period. Ideally, the trend line slope should have been positive during the low mental effort stage (although due to no prechange data being collected, we cannot know how much this would need to have increased). This reverse effect may reflect John's incomplete compliance with the intervention. For instance, John did not always practice in front of the net, nor did he adhere to the tapering schedule designed for him. Unfortunately, John generally struggled to commit to the stage approach, in part due to his desire to maintain previous performance standards. Worryingly, however, once the target variable reached its intended position, John retrospectively reported switching focus to other kinematic variables during practice, explaining that they were moving "incorrectly." Failure to fully reautomate technique before evaluating the skill's entirety would, therefore, unsurprisingly result in the need to reapply conscious attention to the target variable in a cyclical strategy. Accordingly, we suggest this to have caused the slope direction not to have changed; although, the increase in level demonstrates some ability to reduce conscious control on occasions. Moving forward, the next case study provides a more complete account of progress with closer reference to the Five-A Model.

\section{Case Study 2: Chris "the Better"}

\section{Background of Individual and Technique}

Chris had worked with several different coaches over the past 5 years in an attempt to refine his swing plane during the downswing, unfortunately with no long-term success. Despite being a long hitter of the ball, Chris had a natural tendency to overdraw or "hook" some of his shots (i.e., the ball flight curved too much from right to left); the refinement was therefore intended to achieve a straighter ball flight which was explained to be an important aspect to enabling his competitive performances to improve. With the golf season in its latter stages, it was decided between Chris, the coach, and first author that benefit would come from trying a new and structured approach to refining Chris' technique during the forthcoming off season. As such, an initial application of the Five-A Model was attempted. 


\section{Procedures Used in Case Study 2}

The intervention followed recommendations of the Five-A Model; for a comprehensive breakdown of each stage, see Carson and Collins (2011, pp. 158-162). Within these five stages, essential characteristics are highlighted below.

Analysis. Data were collected to establish Chris' technique and levels of functional variability. The required refinement was confirmed after several observations during practice and play, assessed using standard coaching procedures. Crucially, Chris discussed his perceptions of practice behavior, performance, and previous coaching experiences; therefore affording the presentation of the Five-A Model principles against a personally relevant background. Detailed consideration of the complexity required to refine technique helped develop a sense of trust and motivation to improve (i.e., Chris understood why he was to employ certain training procedures); psychological and practice aspects were reportedly novel.

Awareness. Conscious attention during the downswing was raised using kinaesthetic and visual imagery while hitting in front of a net and undertaking a random practice schedule. Video footage of Chris' original and new version swing were replayed on an electronic screen (Apple Inc., USA) while performing contrast drills (consisting of an alternating practice schedule between an already existing "incorrect" or "old" and desired "correct" or "new" movement pattern; cf. Collins, Morriss, \& Trower, 1999; Hanin, Korjus, Jouste, \& Baxter, 2002). As such, physical practice sessions were carried out simultaneously with mental simulation using old and new version self-models.

Adjustment. Chris continued to employ mental imagery; however, the observed and kinaesthetic stimuli were progressively modified, based on best attempts (cf. Carson, Collins, \& Jones, 2014). Chris also gradually tapered out the contrast drills and practice in front of the net. Accordingly, reducing the number of old skill versions increased the requirement to execute his new and desired technique. Gradually reintroducing environmental stimuli by practicing on the driving range, and later the golf course, was intended to prevent too quick a return to automaticity/low mental effort conditions (as explained in Case Study 1).

(Re)Automation. Following consistent demonstration of the newly refined technique, a holistic rhythm-based cue was incorporated into the visual and kinaesthetic imagery script. Similarly to the footfall bleeps introduced by Collins et al. (1999) in Olympic javelin throwing, the cue summarized the preshot routine and swing execution, acting as a "source of information" (MacPherson et al., 2008, p. 289). Difficult but realistic (as judged by Chris) performance and process goals were set, reflecting standards against the previous two seasons' statistics for golf shot accuracy and his recent form.

Assurance. Once Chris had reported a consistent level of comfort in using the new mental simulation routine, he was subjected to competitively simulated environments. This was achieved by combining physical exertion with technically difficult task demands, using a cycle ergometer on the driving range and short sprints on the golf course. The inclusion of added anxiety (increased heart and breathing rate) and the ability to provide feedback, both qualitative (via 
immediate video review) and quantitative (by means of kinematic information), were important facets of this final stage, as a way of convincing both Chris and his coach that the refinement was secure and therefore should not be consciously returned to. Performance feedback and a debrief with the coach and Chris was used to yet further refine the imagery script to include feelings of physiological "readiness" in combination with the various mental strategies.

\section{Data Analysis of Case Study 2}

The target variable was defined by referencing the left hand position to the right elbow in the anterior-posterior axis, the nontarget variable as the left elbow position to the sternum in the medial-lateral axis. Data $\left(N_{\text {trials }}=390\right)$ were taken from a "mid-downswing" event defined as the frame when the left hand crossed a threshold of $0.0 \mathrm{~m}$ relative to a predetermined position on the spine (VT12L3) in the local vertical axis on swing decent.

\section{Results of Case Study 2}

Data for Chris (Figure 2) are divided into the five intervention stages, with follow up tests on Days 208 (28 days) and 257 (77 days).

Chris showed an initial tendency to exert mental effort ( 75; Figure 2A). Through direct questioning, it was established that this cognition related to trying to increase weight shift during the downswing. Advice to try and swing with a natural (less consciously controlled) technique, resulted in Chris confirming that he understood the Analysis stage's aim; mental effort subsequently decreased to a lowest score of 5 . The Awareness stage was characterized by a rapid and then gradual increase in mental effort toward the target variable (Analysis $\Delta$ slope $=$ $\times 0.84$ ), reaching a maximum rating of 138 (Analysis $\Delta$ level $=\times 4.2$ ). Subsequently, mental effort reduced by approximately half of that during the Adjustment stage ( $\Delta$ level $=\div 1.62$ ), in addition to a reversal in trend line slope $(\Delta$ slope $=\div 0.087)$. (Re)automation did not occur immediately; mental effort gradually reduced at this stage, although at a faster rate compared with the Adjustment stage $(\Delta$ slope $=$ $\div 3.22$ ) and, importantly, in the opposite direction to the Awareness stage ( $\Delta$ slope $=\div 0.28$ ). Chris' lowest rating was 10 within the Assurance stage, which showed an insignificant difference $(p=.5)$ at the Follow up stage. Significant differences $(p<.05)$ were evident between the Analysis and all other stages apart from Assurance and Follow up, the same was true for the Awareness stage. There were also no significant difference between Adjustment and (Re)Automation $(p=.16)$ or (Re)Automation and Assurance ( $p=.063)$ stages, which is unsurprising due to the negative trends.

During the Analysis stage, target variable intersession variability was small, showing a mean level of $31.74 \mathrm{~cm}$ (Figure 2B). Intersession variability increased during the Awareness stage $($ range $=30.5 \mathrm{~cm})$ with only a small change in level from the Analysis stage $(\Delta$ level $=\times 1.03)$; crucially, however, the trend line slope reversed its direction in favor of the intended refinement $(\Delta$ slope $=\times 0.008)$. No significant difference in kinematics were found between these two stages, $p=.94$. The Adjustment stage produced more consistent and desirable kinematics compared with the Analysis stage $(\Delta$ level $=\times 1.44)$, as Chris became familiar with the 

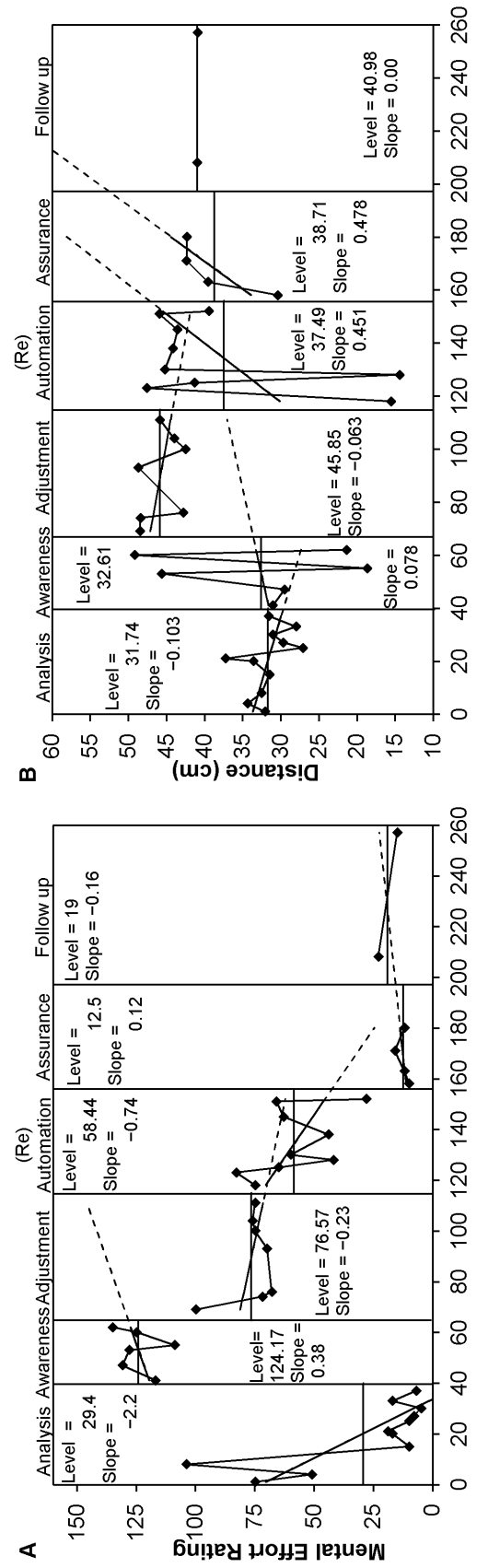
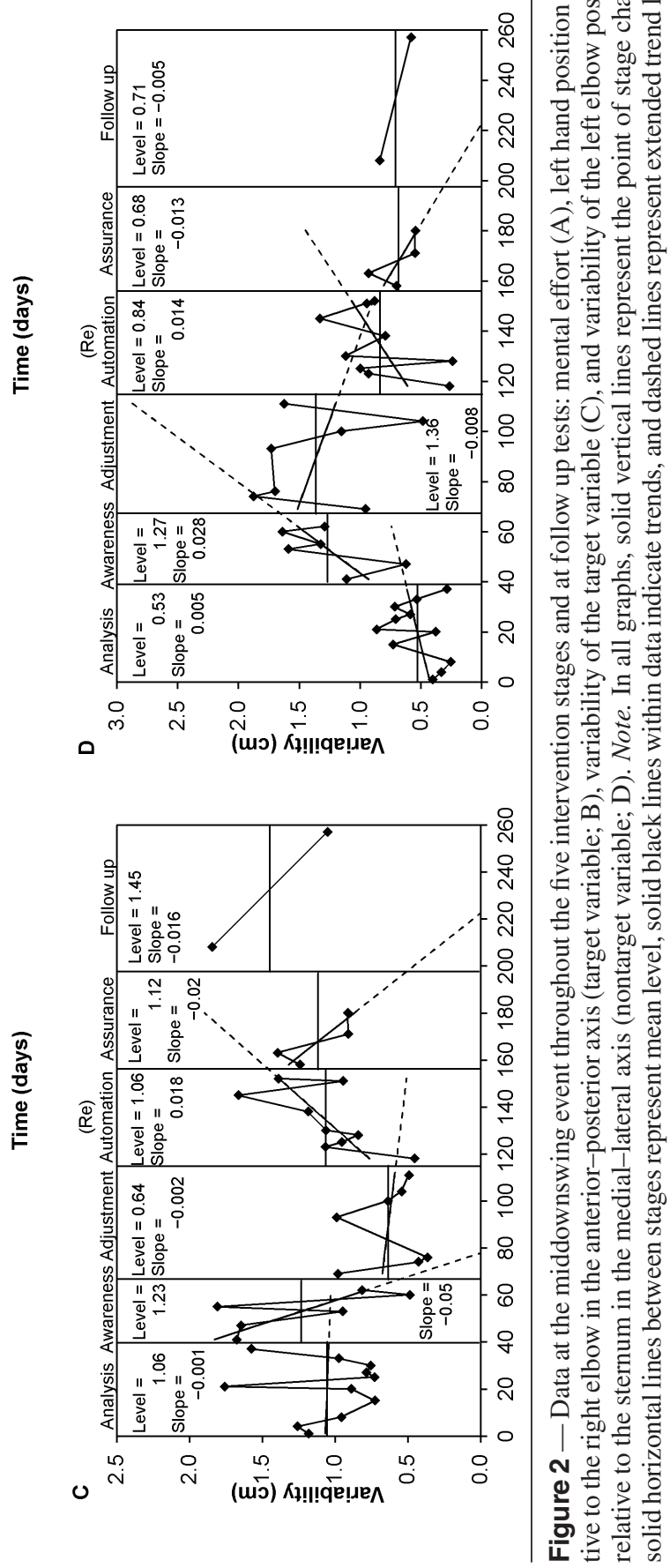
movement and training procedure (cf. variability levels during this stage). Indeed, the Binomial test revealed significant differences between the Analysis and Awareness stages to that of the Adjustment stage, $p=.008$. Introduction of new mental skills during $(\mathrm{Re})$ Automation resulted in a reduction in level $(\Delta$ level $=\div 1.22)$, but was still in the favored direction compared with the Analysis stage $(\Delta$ level $=\times 1.18)$. Attesting to the long-term security of the refinement, no significant differences were found between the Adjustment and (Re)Automation $(p=.25)$ nor Assurance $(p=.375)$ stages; notably, however, the trend line slopes remained positively away from the original kinematics (e.g., Analysis to Assurance stage $\Delta$ slope $=\times 0.05$ ). Accordingly, after Day 128 the technique remained consistent across sessions with a gradually increasing level over the remainder of the intervention and Follow up stage. Technique remained at the newly established position and was also evident at Follow up tests (Analysis $\Delta$ level $=\times 1.29$ ).

Movement variability largely support the patterns expected (Figure 2C). For the target variable the level unexpectedly increased from the Analysis to Awareness stage $(\Delta$ level $=\times 1.2)$; however, the slope steepened in a negative direction $(\Delta$ slope $=$ $\div 50$ ). From visual inspection, it is clear that deautomation did not happen in a linear fashion, perhaps helping to explain this stage's kinematic data. Variability levels were at their lowest during the Adjustment stage ( $\Delta$ Analysis and $(\mathrm{Re})$ Automation level $=\div 1.66$ ), with a predictably shallow slope compared with the Awareness stage $(\Delta$ slope $=\times 25)$. Crucially, the level at Analysis and $(\mathrm{Re})$ Automation stages were exactly the same. Consistent increases in level from the Adjustment to Follow up stages $(\Delta=\times 2.3)$ further indicate the lessening application of conscious attention. Indeed, and in contrast to the Awareness stage, the (Re)Automation stage showed a positive trend $(\Delta$ Awareness slope $=\times 0.001)$. However, despite an increase in level between the (Re)Automation to Assurance stage, the slope reversed direction ( $\Delta$ slope $=\div 0.0004$ ). Contrary to this trend, follow up data demonstrate a continued tendency toward increased variability levels. Accordingly, changes in level, as opposed to slope, may be a better single indicator of progress. Binomial tests showed significant differences between key stages of Analysis and Adjustment ( $p$ $=.008)$, Awareness and Adjustment $(p=.008)$, Awareness and $(\mathrm{Re})$ Automation $(p=.002)$, and Adjustment and (Re)Automation $(p=.018)$, all other comparisons yielded insignificant differences $(p>.05)$. Notably, insignificant differences were expected between Analysis and (Re)Automation and Assurance and Follow up stages. We predict that significant differences were not found in expected cases between Awareness and Adjustment with reference to Assurance and Follow up stages due to the low number of sessions included within these latter stages.

The nontarget variable also largely supports the predicted variability pattern (Figure 2D). Levels increased from the Analysis to Awareness stage $(\Delta$ level $=$ $\times 2.4$ ), with only a slightly higher level in the Adjustment stage (Analysis $\Delta$ level $=$ $\times 2.7)$, before returning back toward Analysis stage levels in the (Re)Automation $(\Delta$ level $=\times 1.6)$, Assurance and Follow up $(\Delta$ level $=\times 1.3)$ stages. As such, change for this swing parameter is pronounced; systematically increasing and then decreasing throughout the refinement process. Trend lines also increased in slope between the Analysis and Awareness stage $(\Delta$ slope $=\times 5.6)$, reversing to a shallower and negative slope in the Adjustment stage (Awareness $\Delta$ slope $=\div 0.002$ ). Apart from the (Re)Automation stage, which demonstrated a positive trend, remaining trend line 
slopes were expectedly negative, with the Follow up showing a shallower slope compared with the Assurance stage $(\Delta$ slope $=\times 2.6)$. Binomial tests show the same results as the target variable at the level of significance, with exception from the Adjustment to $(\mathrm{Re})$ Automation stage $(p=.16)$.

\section{Brief Discussion of Case Study 2}

Chris' data provide a detailed examination of technical refinement using motor control and kinematic measures. Data support the nonlinear mechanism of refinement and, therefore, need for frequent measurement. Despite the degree of "noise" within these data, perhaps as a consequence of the applied testing conditions, movement variability, at least, can be seen to reflect a useful indicator of control, demonstrating support for the patterns predicted by Carson, Collins, et al. (2014a).

Reflecting the target variable kinematics (Figure 2A), data could be interpreted to provide a useful insight into the experiences of skilled performers when attempting technical refinements. Notably, high intersession variability during Awareness and (Re)Automation stages indicate highly "turbulent" times. Increased intersession variability can no doubt be a source of great frustration, supporting the need for committed performers who possess sufficient mental skill to negotiate the refinement pathway. In this case, previously unsuccessful efforts to refine technique led Chris to being open-minded and committed to trying new ideas; reported continuously throughout the intervention between Chris and the corresponding author, particularly when addressing the use of mental skills and practice structure. Constant contact with Chris also helped reassure him during these less consistent times that his experiences were expected. Indeed, during the Analysis stage this was presented to him using schematics of the refinement process.

The following case study attempted to replicate the same effects with a different performer and technical refinement, to test the tracking measures for further validity.

\section{Case Study 3: Peter "the Ugly"}

\section{Background of Individual and Technique}

Peter was a competitive amateur golfer who was dedicated to practicing and trying to improve. However, Peter's coach felt that his rate of improvement was slowing and a clear route forward was difficult to find. Primarily, the coach reported too little focus on processes which underpin performance gains; too much emphasis was placed on the shot outcome and immediate consequences within practice and play. Ultimately, there was limited shared understanding of how Peter would gain long-term benefit. Peter's predominant ball flight was a fade (left to right ball flight) which, the coach considered as a limitation to his performance; a draw shot (right to left ball flight) was preferred. Therefore, the first author was contacted by the coach during the offseason to implement a technical refinement, requiring Peter to "drive" his hips laterally toward the target. Consequently, this was intended to alter the swing path during the downswing and create the conditions necessary to implement a draw shot. 


\section{Procedures Used in Case Study 3}

Since the aim of this case study was to replicate the findings of Case Study 2, the procedures employed were comparable, only this time related to Peter and his intended refinement. Unfortunately, due to Peter's difficulty in engaging with the intervention design and in particular the use of mental skills (see Brief Discussion of Case Study 3), data collection ceased in what was intended to be the Awareness stage.

\section{Data Analysis of Case Study 3}

Considering the unsuccessful nature of this intervention, a target and nontarget variable could not be clearly discerned. Reflecting Peter's intended technical refinement, a target variable of the left shoulder position was referenced to the pelvis in the medial-lateral axis. Presenting a myriad of unrelated swing parameters which too did not conform to the expected pattern of an increase in variability, the left hand position referenced to the sternum in the medial-lateral axis is included as an exemplar of this unintended effect. Data $\left(N_{\text {trials }}=280\right)$ were extracted from the same mid-downswing event as described in Case Study 2.

\section{Results of Case Study 3}

Data for Peter (Figure 3) are divided into three sections, representing preintervention, intervention, and follow up stages. Peter did not continue past the Awareness stage and kinematic data do not reflect the expected patterns. Accordingly, stages presented in Figure 3 are labeled generically and not in correspondence to the Five-A Model. This inability to complete the intervention was in part as a result of prolonged uncertainty over using the recommended mental skills; nor was Peter totally convinced of the technical aspect requiring refinement. Throughout the intervention period, this manifested itself as a constant switching between technical components and evaluation over a short-term time scale. Despite this confusion, mental effort (Figure 3A) was reportedly low and decreasing during the preintervention stage, which significantly increased, $p<.001$, during the intervention period $(\Delta$ level $=$ $\times 7.1, \Delta$ slope $=\times 2)$, remaining high throughout. Follow up tests on Days 177 (31 days) and 233 (87 days) show a reduction to a lowest score of 0 ; however, Binomial tests found no significant difference to either preintervention $(\Delta$ level $=\times 2.4, \Delta$ slope $=\div 13.33$ ) or intervention $(\Delta$ level $=\div 2.9, \Delta$ slope $=\div 26.67)$ stages, $p=.25$.

While target variable kinematics (Figure 3B) demonstrate a slight change in position from the pre- to early sessions of the intervention stage, by approximately $5 \mathrm{~cm}$, subsequent sessions also reveal that this was not maintained ( $\Delta$ level $=\div 2.9$ ); a clear and consistent regression back to the original kinematics are evident from Day $135(\Delta$ slope $=\times 2)$, which remained during follow up tests (preintervention to follow up $\Delta$ level $=\div 2.25, \Delta$ slope $=\div 0.0001$ ). Variability levels show little difference for the target variable across the three stages (levels $=1.2,1.32$, and 1.14). Despite a change in slope direction between preintervention and intervention stages $(\times 0.000014)$, preintervention and follow up stages $(\times 0.00001)$, and a reduction in slope between intervention and follow up stages $(\div 1.4)$, insignificant 

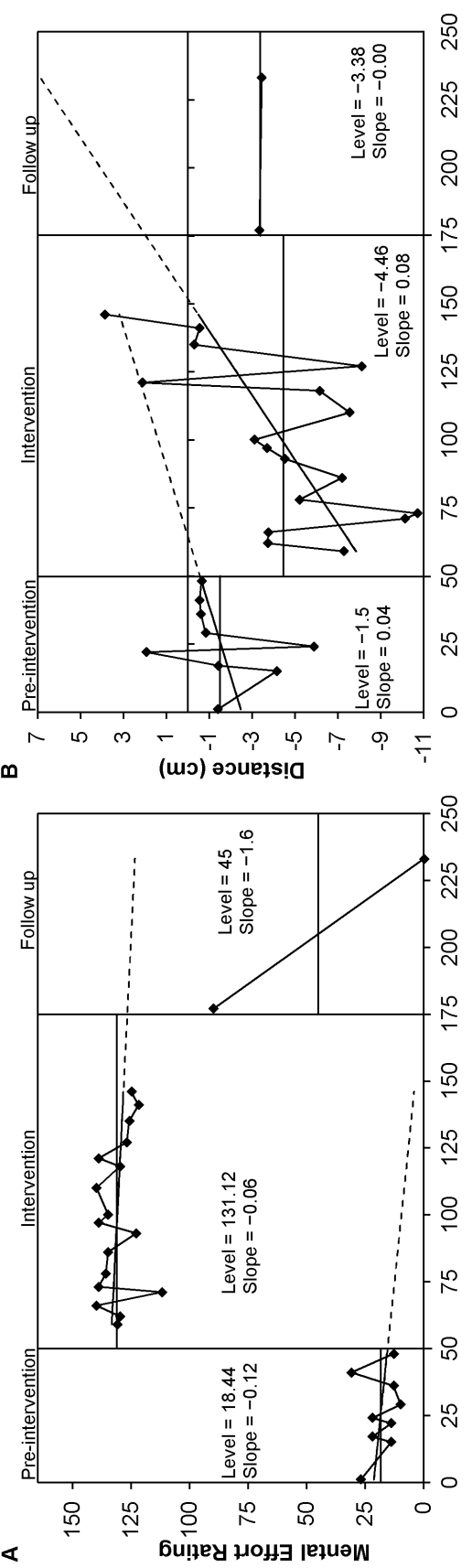

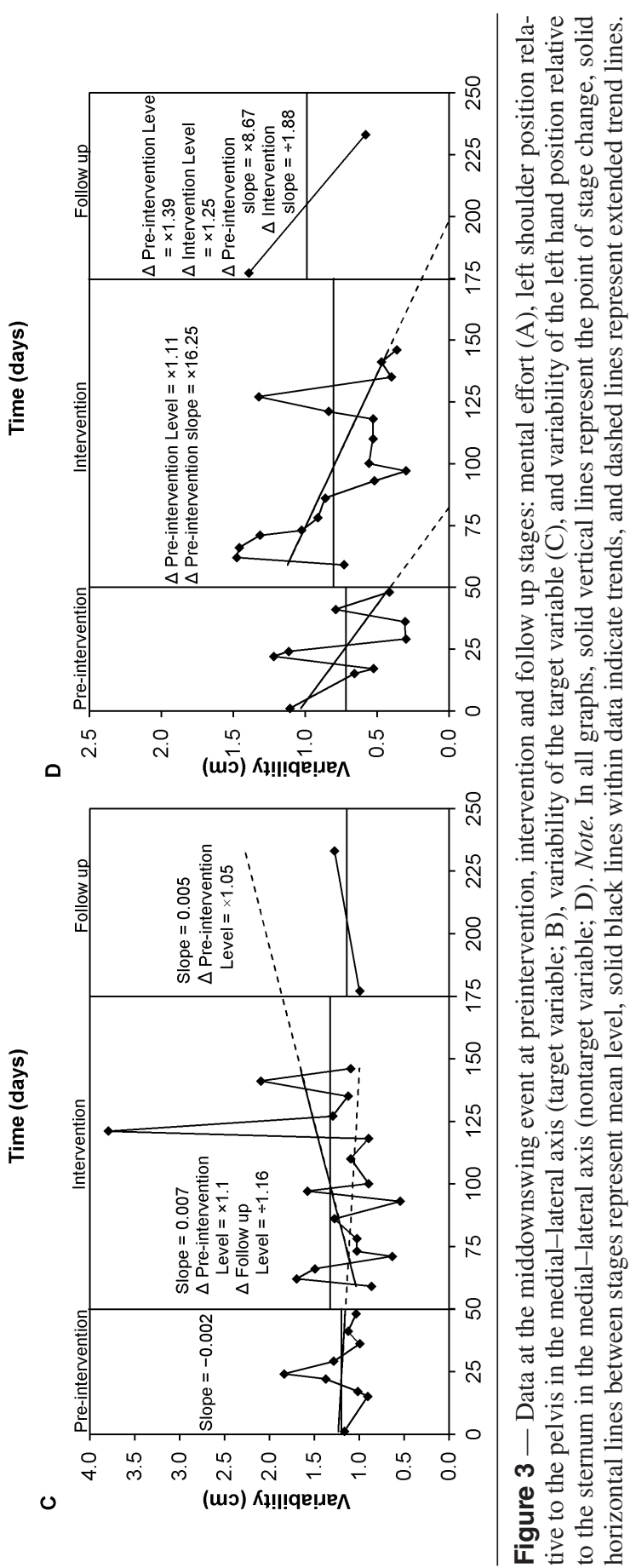


Binomial test results (preintervention to intervention, $p=.15$; preintervention and intervention to follow up, $p=.25$ ) further suggest little to no difference in motor control. This seems even more evident when considering the outlier on Day 121. The nontarget variable showed a consistently negative trend in all stages $(-0.13$, -0.008 , and -0.015$)$, despite systematic increases in level $(0.72,0.8$, and 1$)$. There was a significant difference between preintervention and intervention stages, $p<$ .001 , but not between either of these stages and follow up, $p=.25$.

Importantly, a limitation of the split-middle technique is that it assumes no limits to achieving the predicted trend (cf. Shambrook \& Bull, 1996); whereas, variability cannot be negative (or probably even zero for this type of task), which was predicted in this case study and Case Study 2 (see Figure 2C and 2D). As already mentioned, while statistical analyses (even simple ones) might appear to add objectivity to interpreting data, we recommend readers to consider what would constitute a meaningful rather than statistical difference between intervention phases, with the pattern of trends being the main focus.

\section{Brief Discussion of Case Study 3}

This case study was unfortunately unsuccessful in terms of delivering a refinement to Peter's technique. As such, data are provided to inform applied coaching practice about what to avoid (see also Rendell, Farrow, Masters, \& Plummer, 2011). Crucially from this case study, is the strong influence that Peter's mind-set had on the process. Peter had not "bought into" the identified technical component as being in need of refinement, nor did he feel confident in his ability to employ the mental skills being administered; undoubtedly as a result of inexperience in using them. Peter was also very expressive about the novelty of approach taken. Through fascination of the methods, Peter explicitly contrasted these with his previous coaching, resulting in a self-reported state of confusion and over analysis. Consequently, for these reasons, the inconsistent movement variability data, and shortly approaching competitive season, it was decided and agreed to abort the refinement process during a detailed debrief with both Peter and the coach. In short, we did not believe that Peter could fully refine his technique before the first competition and/or, given the presenting complications, with sufficient time to benefit during the upcoming competitive season.

Addressing the movement variability data, it is probably due to Peter's inexperience of using specific mental skills and short-term experimental approach toward improving his technique that the expected variability patterns did not emerge. Importantly, however, even if Peter consistently employed such an approach, it is questionable whether the required measures of functional variability (i.e., performing with a largely subconscious level of control) were in fact established; signifying an element of self-report/presentation bias when completing the RSME before initiating the intervention. From a theoretical perspective, evidence that the technique regressed back toward the original version is supportive of previous findings, which attest the relative permanency of technique at this high level (e.g., MacPherson, Turner, \& Collins, 2007) and thus, highlight the need for a distinct systematic process to that of skill acquisition when implementing refinements (Carson \& Collins, 2011). 


\section{General Discussion}

The aims of this study were to provide an initial check of precepts pertaining to the Five-A Model and examine the dynamic interaction between coaching, psychomotor, biomechanical, and psychological inputs to the skill refinement process. The case study data provide several important contributions to the literature and coaching practice. A consistent underlying factor of success was the influence of psychobehavioral factors. For instance, John did not adhere to some aspects of practice and Peter struggled to employ the mental skills which resulted in states of confusion (cf. Prochaska \& Prochaska, 1999). Paradoxically, Chris worked hard to develop his mental skills and adhered fully to the training design. As such, we believe data were able to show this influence-reflecting John's "double-dip," Peter's "flat" and Chris' largely expected change in variability patterns. This finding complements those from talent development research, which has identified pertinent psychobehavioral factors as crucial to long-term competitive success; for example, the Psychological Characteristics of Developing Excellence (PCDEs; MacNamara, Holmes, \& Collins, 2008). Within these characteristics are constructs that were highly relevant to the case studies (e.g., imagery, realistically evaluating performance, self-regulation, and commitment) and are also highlighted by Carson and Collins (2011) within the Five-A Model. Consequently, PCDEs (or at least a revision to match usage by more mature/senior athletes) form a crucial component to processes involving the development of talent and technical refinement. Since both processes are longitudinal in nature - albeit successful refinement will probably last months, whereas talent development years-athlete commitment to multiple training components is vital. Accordingly, those performers already possessing these skills are most likely to be successful at making a long-term permanent and pressure resistant technical refinement within a timely duration. High-level coaching should, therefore, be considered as that which proactively "equips" performers with experience, if not mastery, of these skills before refining technique as a necessity to limiting the risk involved.

Notably, PCDEs impact on both psychomotor and psychosocial skills; the latter being of high importance when entering the Analysis stage where, in the cases of John and Peter, this was not conducted to optimal effect. In fact, incorrectly diagnosing a target variable as being in need of refinement, or at least disagreement between player and coach (as was evident in Case Study 3), is also worth highlighting as a potential risk involved when implementing technical refinements. The additional absence of well-developed psychomotor skills in these case studies could be interpreted as a reflection on participants' previous experience during skill acquisition. Offering support to this suggestion, all participants expressed use of the mental skills as novel. Furthermore, this finding corroborates those of Carson et al. (2013) which show high-level amateurs and European Tour professionals to infrequently employ mental skills, at least with intent, and coaches not always attending to factors such as "buy in." The cyclical nature of technical refinement reported by European Tour professionals and coaches is similar to Case Study 1. Therefore, based on the current study and that of Carson et al. (2013), there is a clear and current need within golf to improve coach and player education regarding the fundamental benefits of acquiring intentional mental skills, alongside the 
development of appropriate practice structure and training environments. Since there is little evidence of this emerging from the golf coaching literature, and the evidenced lack of consistent comprehension in this area, it would seem that consultation with applied sport scientists presents a viable solution to resolving this issue (R. Martindale \& Nash, 2012). Of course, such a partnership would also rely on coaches being receptive, open to innovation, willing to learn, and change some of their coaching behaviors (Collins, Abraham, \& Collins, 2012).

In assessing the quality of kinematic and variability data, there was a large amount of noise, or intersession variability. This is probably an inherent result of conducting analyses within applied settings and over long-term time scales. Indeed, since data suggest variability and cognition to be linked, noise may exist as a direct result of the constantly changing constraints (e.g., ambient temperature, mood, and energy levels) imposed on the performer (Newell, 1986). Crucially, a low-level ability to psychologically respond to such perturbations would result in less consistent states of control. Accordingly, for performers that are highly-trained and committed to using mental skills, one might expect to see less intersession variation. We would therefore suggest that frequent data collection can overcome this issue, especially when attempting to track a nonlinear process such as technical refinement. Resulting from frequent data collection, measures employed in these case studies appear to provide useful "psychomechanical" data to guide coaches' decision making. A key message here is to be able to recognize when a refinement is, or is not, following the expected plan, what needs to and can be done to resolve any issues, and when to abort a technical refinement before the desired outcomes become too problematic. As such, these case studies offer equally as informative guidance on what to avoid (i.e., a double dip or flat progression) as well as what to aim for.

Considering the requirement for pressure resistance at this elite-level of performance, we acknowledge a limitation of these case studies by not reporting in-depth interventions and effects designed to achieve this. Rather, our primary intention was to report on the process in its entirety. Such study is, however, a feature of our planned future work in golf and other sporting domains. Indeed, the need for future research in this area can be evidenced by findings that internationally competitive amateur golfers employ technical modifications during performance as a coping strategy under pressure (Nicholls, Holt, Polman, \& James, 2005). Contrasted against existing theory (e.g., Masters, 1992) and practice (e.g., MacPherson et al., 2008), such a strategy appears counterproductive if intended to promote optimal skill execution.

Finally, we welcome future research on the topic of refining and regaining skills within related domains of performance; namely, rehabilitation. Carson, Collins, and Jones (2014) have provided a detailed case study of an Olympic weightlifter successfully returning from injury through utilization of methods advocated within the Five-A Model; however, without the inclusion of intraindividual movement variability data. Within the clinical setting, future work could explore how the Five-A Model, or its constituent parts, might contribute to services provided by physiotherapists and healthcare professionals working with patients recovering from musculoskeletal injury and joint replacement. A review of the physiotherapy profession shows definite inclusion of physiological, neurological, and biomechanical aspects of therapy, although reported psychological aspects mainly reflect 
counseling skills as opposed to psychomotor processes during execution (Higgs, Refshauge, \& Ellis, 2001). As such, we believe it is from this perspective and the provision of a theoretically grounded and systematic process that services might be enhanced.

In conclusion, we have provided evidence to demonstrate how long-term success in refining an already learnt and well-established skill can be optimized using a systematic approach and tracked with a variety of measures. Individual analyses within elite-level golf showed specific practice design, combined with mental skills, to result in causative effects on movement variability. Data support suggestions from previous research (e.g., Carson, Collins, et al., 2014a) linking cognition and movement variability such that, a coach is able to recognize changes in kinematics and levels of automaticity against predetermined effects. Crucially, at a practical level, characteristic hallmarks of successful and unsuccessful progress were highlighted. Specifically, there is a strong need to consider the nature of the change and that this is effectively sold to the performer. If the benefit is not understood, it is unlikely that performers will execute with maximum intention or be committed over the necessary timescale. Furthermore, it is important to tailor practice environments to encourage changes in automaticity during this nonlinear process. Finally, coaches must work to individualize the training practices and employment of mental skills advocated within the Five-A Model, perhaps even upskilling/educating their performers as a proactive step. We hope that this study will be used by coaches to better understand the complex cognitive-co-ordinative (cf. Schack \& Bar-Eli, 2007) process of skill refinement and guide effective decision making.

\section{Note}

1. Although we contextualize this paper within elite sport, the concepts presented are also applicable to performers experiencing arrested expertise, whereby performance has stabilized at a sub elite standard.

\section{Acknowledgment}

We wish to thank Dr. Caroline Wakefield for her assistance with the analysis of data.

\section{References}

Bernstein, N.A. (1967). The coordination and regulation of movements. Oxford: Pergamon Press.

Bjork, E.L., \& Bjork, R.A. (2011). Making things hard on yourself, but in a good way: Creating desirable difficulties to enhance learning. In M.A. Gernsbacher, R.W. Pew, L.M. Hough, \& J.R. Pomerantz (Eds.), Psychology and the real world: Essays illustrating fundamental contributions to society (pp. 56-64). New York: Worth Publishers.

Brown, S.J., Selbie, W.S., \& Wallace, E.S. (2013). The X-Factor: An evaluation of common methods used to analyse major inter-segment kinematics during the golf swing. Journal of Sports Sciences, 31, 1156-1163. PubMed doi:10.1080/02640414.2013.775474

Callow, N., Hardy, L., \& Hall, C. (2001). The effects of a motivational general-mastery imagery intervention on the sport confidence of high-level badminton players. Research Quarterly for Exercise and Sport, 72, 389-400. PubMed doi:10.1080/02701367.200 1.10608975 
Carson, H.J., \& Collins, D. (2011). Refining and regaining skills in fixation/diversification stage performers: The Five-A Model. International Review of Sport and Exercise Psychology, 4, 146-167. doi:10.1080/1750984X.2011.613682

Carson, H.J., \& Collins, D. (2014). Effective skill refinement: Focusing on process to ensure outcome. Central European Journal of Sport Sciences and Medicine, 7, 5-21.

Carson, H.J., \& Collins, D. (2016).The fourth dimension: A motoric perspective on the anxiety-performance relationship. International Review of Sport and Exercise Psychology, 9, 1-21. doi:10.1080/1750984X.2015.1072231

Carson, H.J., Collins, D., \& Jones, B. (2014). A case study of technical change and rehabilitation: Intervention design and interdisciplinary team interaction. International Journal of Sport Psychology, 45, 57-78. doi: 10.7352/IJSP.2014.45.057

Carson, H.J., Collins, D., \& MacNamara, Á. (2013). Systems for technical refinement in experienced performers: The case from expert-level golf. International Journal of Golf Science, 2, 65-85.

Carson, H.J., Collins, D., \& Richards, J. (2014a). Intra-individual movement variability during skill transitions: A useful marker? European Journal of Sport Science, 14, 327-336. PubMed doi:10.1080/17461391.2013.814714

Carson, H.J., Collins, D., \& Richards, J. (2014b). "To hit, or not to hit?" Examining the similarity between practice and real swings in golf. International Journal of Golf Science, 3, 103-118. doi:10.1123/ijgs.2014-0003

Carson, H. J., Collins, D., \& Richards, J. (2015). Initiating technical refinements in high-level golfers: Evidence for contradictory procedures. European Journal of Sport Science, Advance online publication. doi:10.1080/17461391.2015.1092586

Christina, R.W., \& Corcos, D.M. (1988). Coaches guide to teaching sport skills. Champaign, IL: Human Kinetics.

Collins, D., Abraham, A., \& Collins, R. (2012). On vampires and wolves - Exposing and exploring reasons for the differential impact of coach education. International Journal of Sport Psychology, 43, 255-272.

Collins, D., Morriss, C., \& Trower, J. (1999). Getting it back: A case study of skill recovery in an elite athlete. The Sport Psychologist, 13, 288-298.

Fitts, P.M., \& Posner, M.I. (1967). Human performance. California: Brooks/Cole Publishing Company.

Gentile, A.M. (1972). A working model of skill acquisition with application to teaching. Quest, 17, 3-23. doi:10.1080/00336297.1972.10519717

Glenberg, A., Schroeder, J., \& Robertson, D. (1998). Averting the gaze disengages the environment and facilitates remembering. Memory \& Cognition, 26, 651-658. PubMed doi:10.3758/BF03211385

Hanin, Y., Korjus, T., Jouste, P., \& Baxter, P. (2002). Rapid technique correction using old way/new way: Two case studies with Olympic athletes. The Sport Psychologist, 16, 79-99.

Higgs, J., Refshauge, K., \& Ellis, E. (2001). Portrait of the physiotherapy profession. Journal of Interprofessional Care, 15, 79-89. PubMed doi:10.1080/13561820020022891

MacNamara, Á., Holmes, P., \& Collins, D. (2008). Negotiating transitions in musical development: The role of psychological characteristics of developing excellence. Psychology of Music, 36, 335-352. doi:10.1177/0305735607086041

MacPherson, A.C., Collins, D., \& Morriss, C. (2008). Is what you think what you get? Optimizing mental focus for technical performance. The Sport Psychologist, 22, 288-303.

MacPherson, A.C., Collins, D., \& Obhi, S.S. (2009). The importance of temporal structure and rhythm for the optimum performance of motor skills: A new focus for practitioners of sport psychology. Journal of Applied Sport Psychology, 21, 48-61. doi:10.1080/10413200802595930 
MacPherson, A.C., Turner, A.P., \& Collins, D. (2007). An investigation of natural cadence between cyclists and noncyclists. Research Quarterly for Exercise and Sport, 78, 396-400. PubMed doi:10.5641/193250307X13082505158426

Marlow, C., Bull, S.J., Heath, B., \& Shambrook, C.J. (1998). The use of a single case design to investigate the effect of a pre-performance routine on the water polo penalty shot. Journal of Science and Medicine in Sport, 1, 143-155. PubMed doi:10.1016/S14402440(98)80010-8

Martindale, A., \& Collins, D. (2007). Enhancing the evaluation of effectiveness with professional judgment and decision making. The Sport Psychologist, 21, 458-474.

Martindale, R., \& Nash, C. (2012). Sport science relevance and application: Perceptions of UK coaches. Journal of Sports Sciences, 31, 807-819. PubMed doi:10.1080/0264 0414.2012 .754924

Masters, R.S.W. (1992). Knowledge, knerves and know-how: The role of explicit versus implicit knowledge in the breakdown of a complex motor skill under pressure. British Journal of Psychology, 83, 343-358. doi:10.1111/j.2044-8295.1992.tb02446.x

Mesagno, C., \& Mullane-Grant, T. (2010). A comparison of different pre-performance routines as possible choking interventions. Journal of Applied Sport Psychology, 22, 343-360. doi:10.1080/10413200.2010.491780

Newell, K.M. (1986). Constraints to the development of coordination. In M.G. Wade \& H.T.A. Whiting (Eds.), Motor development in children: Aspects of coordination and control (pp. 341-360). Dordrecht, The Netherlands: Martinus Nijhoff. doi:10.1007/97894-009-4460-2_19

Newell, K.M., Liu, Y-T., \& Mayer-Kress, G. (2001). Time scales in motor learning and development. Psychological Review, 108, 57-82. PubMed doi:10.1037/0033-295X.108.1.57

Nicholls, A.R., Holt, N.L., Polman, R.C.J., \& James, D.W.G. (2005). Stress and coping among international adolescent golfers. Journal of Applied Sport Psychology, 17, 333-340. doi:10.1080/10413200500313644

Prochaska, J., \& Prochaska, J. (1999). Why don't continents move? Why don't people change? Journal of Psychotherapy Integration, 9, 83-102. doi:10.1023/A:1023210911909

Rendell, M.A., Farrow, D., Masters, R., \& Plummer, N. (2011). Implicit practice for technique adaptation in expert performers. International Journal of Sports Science \& Coaching, 6, 553-566. doi:10.1260/1747-9541.6.4.553

Schack, T., \& Bar-Eli, M. (2007). Psychological factors of technical preparation. In B. Blumenstein, R. Lidor, \& G. Tenenbaum (Eds.), Psychology of sport training (pp. 62-103). Münster, Germany: Meyer \& Meyer Sport.

Shambrook, C.J., \& Bull, S.J. (1996). The use of a single-case research design to investigate the efficacy of imagery training. Journal of Applied Sport Psychology, 8, 27-43. doi:10.1080/10413209608406306

Toner, J., \& Moran, A. (2015). Enhancing performance proficiency at the expert level: Considering the role of 'somaesthetic awareness'. Psychology of Sport and Exercise, 16, 110-117. doi:10.1016/j.psychsport.2014.07.006

White, O.R. (1974). The 'split middle': A 'quickie' method of trend estimation. Seattle, WA: University of Washington, Experimental Educational Unit, Child Development and Mental Retardation Centre.

Wood, G., \& Wilson, M. (2011). Quiet-eye training for soccer penalty kicks. Cognitive Processing, 12, 257-266. PubMed doi:10.1007/s10339-011-0393-0

Zijlstra, F.R.H. (1993). Efficiency in work behaviour: A design approach for modern tools. Delft: Delft University Press. 